\title{
Histone Deacetylases in Neural Stem Cells and Induced Pluripotent Stem Cells
}

\author{
Guoqiang Sun, Chelsea Fu, Caroline Shen, and Yanhong Shi \\ Department of Neurosciences, Center for Gene Expression and Drug Discovery, Beckman Research Institute of City of Hope, \\ 1500 E. Duarte Road, Duarte, CA 91010, USA \\ Correspondence should be addressed to Yanhong Shi, yshi@coh.org
}

Received 16 July 2010; Revised 18 May 2011; Accepted 31 May 2011

Academic Editor: Patrick Matthias

Copyright ( 92011 Guoqiang Sun et al. This is an open access article distributed under the Creative Commons Attribution License, which permits unrestricted use, distribution, and reproduction in any medium, provided the original work is properly cited.

Stem cells have provided great hope for the treatment of a variety of human diseases. However, the molecular mechanisms underlying stem cell pluripotency, self-renewal, and differentiation remain to be unveiled. Epigenetic regulators, including histone deacetylases (HDACs), have been shown to coordinate with cell-intrinsic transcription factors and various signaling pathways to regulate stem cell pluripotency, self-renewal, and fate determination. This paper focuses on the role of HDACs in the proliferation and neuronal differentiation of neural stem cells and the application of HDAC inhibitors in reprogramming somatic cells to induced pluripotent stem cells (iPSCs). It promises to be an active area of future research.

\section{Introduction}

Stem cells have the ability to self-renew and differentiate into multiple lineages. Identifying regulators that control stem cell self-renewal and differentiation is essential for the development of stem cell-based cell replacement therapies for human diseases and injuries [1]. Epigenetic control, including histone modification, has been shown to play an important role in regulating both stem cell self-renewal and pluripotency [2-5].

Histone modification by acetylation is the most wellstudied histone modification and has been shown to be an important means of gene regulation [6]. In general, acetylation of histone tail disrupts the electrostatic interaction between positively charged amino acids from the histone tail and negatively charged phosphate group in DNA, leading to decompression of chromatin structure. The open chromatin allows for the access of transcription factors and ultimately gene activation [7]. Acetylated histone tails may also serve as docking sites for the recruitment of bromodomain proteins, a class of transcriptional activator [8]. On the other hand, histone deacetylation leads to gene repression [9]. In addition, transcription factors themselves have also been shown to be regulated by acetylation and deacetylation, respectively [10]. The histone acetylation is mediated by histone acetylases (HATs), while histone deacetylation is catalyzed by histone deacetylases (HDACs). HDACs have been shown to regulate many important biological processes, including cell proliferation, differentiation, and development, by forming complexes with various transcription factors and transcriptional coregulators [8].

Neural stem cells are stem cells of neural origin. They retain the ability to proliferate and self-renew and have the capacity to give rise to both neuronal and glial lineages [11-14]. A complete understanding of neural stem cells and neurogenesis requires the identification of molecules that determine the self-renewal and multipotent character of these cells. These molecules likely include epigenetic regulators, such as HDACs, that act to regulate stem cell selfrenewal and differentiation by controlling the activity of a network of downstream target genes [15].

Recent breakthrough studies using retroviral transduction of a transcription factor quartet to reprogram human somatic cells into induced pluripotent stem cells (iPSCs) have led to an important revolution in stem cell research [16-18]. Comparative analysis of human iPSCs and human embryonic stem cells using assays for morphology, gene expression profiles, epigenetic status, and differentiation 
potential have revealed a remarkable degree of similarity between these two pluripotent stem cell types. These advances in reprogramming will enable the creation of patient-specific stem cell lines to study various disease mechanisms. The cellular models created will provide valuable tools for drug discovery. Furthermore, this reprogramming system provides great potential to design customized patientspecific stem cell therapies with economic feasibility [19]. However, reprogramming by viral infection is a slow process with very low efficiency. Recent progress in using HDAC inhibitors to enhance reprogramming efficiency will be discussed.

\section{HDACs in Neural Stem Cells}

Neural stem cell self-renewal and differentiation are the result of transcriptional control in concert with chromatin remodeling and epigenetic modifications. During central nervous system development in vertebrates, neural stem cell fate is strictly controlled under regional and temporal manners, accompanied by precise epigenetic control [20].

We have shown that HDAC-mediated transcriptional repression is essential for the proliferation and self-renewal of neural stem cells (Figure 1) [21]. There are 11 HDACs in the HDAC superfamily [8]. Among them, HDAC1, HDAC3, HDAC5, and HDAC7 are highly expressed in neural stem cells $[21,22]$. The expression of these HDACs is reduced upon differentiation. On the other hand, HDAC2 expression is more widespread in the brain [22]. While HDAC2 is expressed in proliferating neural progenitors, its expression is upregulated as neurons differentiate [22]. HDAC11 is also predominately expressed in mature neurons and minimally expressed in neural precursors [23].

We showed that HDACs function in neural stem cells through nuclear receptor TLX, an essential neural stem cell regulator [24]. Both HDAC3 and HDAC5 have been shown to be recruited to the promoters of TLX target genes in neural stem cells. Recruitment of HDACs led to transcriptional repression of TLX target genes, the cyclindependent kinase inhibitor p21, and the tumor suppressor gene pten. Disruption of the TLX-HDAC interaction led to substantial induction of p21 and pten gene expression and dramatic inhibition of neural stem cell proliferation [21]. The role of p21 as an HDAC target in cell proliferation was also demonstrated in embryonic stem cells recently [25]. It is worth noting that p 21 has been identified as a target for HDACs in earlier studies as well [26]. While most reports point to p21 as a target for HDACs independent of p53 [27, 28], a direct role for p53 in the induction of p 21 expression has also been reported [29].

In addition to self-renewal, HDACs also regulate neural stem cell differentiation. Treatment of adult neural stem cells with HDAC inhibitors induced neuronal differentiation and upregulated neuronal-specific genes, such as NeuroD, neurogenin 1 (Ngn1), and Math1 (Figure 1) [30-34]. In vitro treatment of adult neural subventricular zone precursor cells with HDAC inhibitors also led to increased production of neurons, with concomitant induction of NeuroD, cyclin D1, and B-lymphocyte translocation gene 3 [33]. In addition to its effect on adult neurogenesis, treatment of embryonic neural stem cells with the HDAC inhibitor trichostatin A (TSA) also led to increased neuronal differentiation, with decreased astrocyte differentiation [30].

Genetic studies using knockout mouse models revealed that HDAC1 is a major deacetylase in embryonic stem cells. HDAC1-null embryonic stem cells exhibit proliferation defects with increased expression of the cyclin-dependent kinase inhibitors p21 and p27 [35]. Furthermore, HDAC1 and HDAC2 play redundant and essential role in the progression of neuronal precursors to neurons. Deletion of both HDAC1 and HDAC2 led to a failure of neuronal precursors to differentiate into mature neurons [36]. On the other hand, HDAC2 is required to silence neural progenitorspecific gene expression during neuronal differentiation in the neurogenic zones of adult brains, as revealed by studies using either conditional deletion of HDAC2 or in mice lacking the catalytic activity of HDAC2 [37].

A wide range of brain disorders have been shown to be associated with the imbalance between the activity of HATs and HDACs [38]. Therefore, HDAC inhibitors have the potential to intervene neurodegenerative diseases. Indeed, HDAC inhibitors have been shown to exhibit neuroprotective effect, induce neurotrophic factor expression, display anti-inflammatory properties, and improve neurological performance in animal models of neurological diseases [38]. Considering the important role of HDACs in controlling neural stem cell proliferation and neurogenesis, HDAC inhibitors may emerge as novel therapeutic tools for the treatment of a variety of neurodegenerative diseases. As a proof-of-principle, the HDAC inhibitor valproate has been used as an anticonvulsant drug and mood stabilizer to treat bipolar disorder, a manic-depressive illness [39].

\section{HDAC Inhibitors in iPSCs}

In addition to neural stem cells that have offered great potential for the treatment of neurological diseases, pluripotent stem cells have also provided great hope for cell replacement therapies for neurological disorders and other degenerative diseases because of their ability to self-renew and their potential to form all cell lineages in the body [40]. Recently, an important revolution in stem cell research has been undertaken. Using a cocktail of four factors, somatic cells can be reprogrammed into iPSCs [16-18, 41-44]. iPSCs are very similar to embryonic stem cells in that both have the capability to self-renew and differentiate into all cell types, but iPSCs are produced from epigenetic reprogramming of somatic cells through the exogenous expression of four pluripotency-related transcription factors $[45,46]$.

iPSCs were first generated by retroviral transduction of four factors, Oct4, Sox2, Klf4, and c-Myc [17, 43]. Since then, the technique has been optimized and conducted in different ways, by selecting the cell types to be reprogrammed, using different combinations of reprogramming genes and improving the method for reprogramming factor delivery [46]. One of the most common reprogramming gene delivery methods is retroviral and lentiviral transduction. However, the efficiency of reprogramming using 


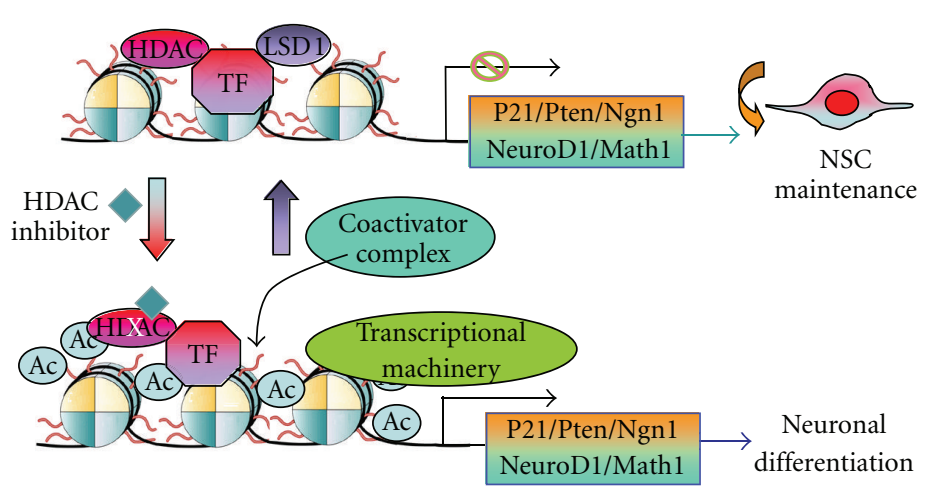

FIGURE 1: HDACs in neural stem cell proliferation and neuronal differentiation. In proliferating neural stem cells (NSCs), transcription factors (TF) recruit HDACs to the promoters of their downstream target genes, to repress the expression of cell cycle inhibitors, such as p21 and pten, and neuronal-specific genes, such as NeuroD, Neurogenin 1 (Ngn1), and Math 1, to maintain NSC proliferation and self-renewal. In addition to promote NSC proliferation, HDACs also inhibit neuronal differentiation. Treatment of HDAC inhibitors leads to induced neuronal differentiation, with increased expression of p21 and pten, and neuronal-specific genes. AC stands for histone acetylation.

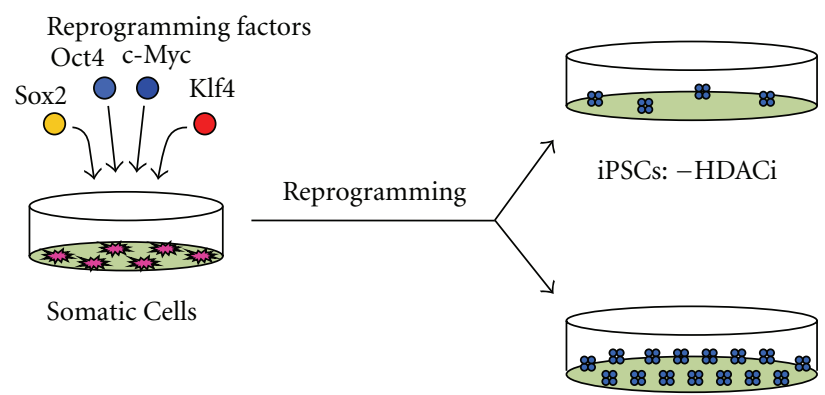

iPSCs: +HDACi

FIgURE 2: Treatment of HDAC inhibitors enhances reprogramming efficiency. When reprogramming somatic cells to iPSCs by ectopic expression of the four transcription factors (Oct4, Sox2, Klf4, and c-Myc), the resulting reprogramming efficiency is low (iPSC: HDACi). But with the use of HDAC inhibitors (HDACi), the reprogramming efficiency can improve by more than 100-fold (iPSC: +HDACi), especially when using the HDACi, valproic acid (VPA). Through this improvement, it is suggested that histone modification plays an important role in inducing pluripotent stem cells.

viral vectors is extremely low and the overall process is slow [47]. Several lines of evidence support the notion that stochastic epigenetic events contribute to the low efficiency of reprogramming [45]. The observation that reprogramming is a slow and gradual process that takes several weeks further supports this notion.

Small molecules involved in epigenetic regulation, such as DNA methyltransferase inhibitors $[48,49]$, histone deacetylase inhibitors $[47,48]$, and histone methyltransferase inhibitors [50], have been shown to improve the efficiency of reprogramming substantially. The histone deacetylases have been shown to enhance the efficiency of reprogramming mediated by somatic cell nuclear transfer up to 5 -fold in a concentration- and time-dependent manner $[51,52]$.

Researchers speculated that reprogramming using defined reprogramming factors may follow a similar mechanism of action to that of reprogramming using somatic cell nuclear transfer. By using an Oct4-GFP reporter gene, scientists determined whether small molecules involved in chromatin modification, such as HDAC inhibitors, played a role in reprogramming mouse embryonic fibroblasts to iPSCs
[48]. Indeed, HDAC inhibitors, including suberoylanilide hydroxamic (SAHA), TSA, and valproic acid (VPA), all increased the efficiency of reprogramming greatly (Figure 2) $[47,48]$. Among these HDAC inhibitors, VPA exhibited the most potent effect and enhanced the reprogramming efficiency more than 100-fold [48]. It is still unclear why VPA effect on reprogramming efficiency is significantly stronger. Suggested ideas include that the toxicity of the other chemicals at tested concentrations may be higher or that VPA may have roles beyond HDAC inhibition [48]. In addition to improving the efficiency of reprogramming four factortransduced mouse embryonic fibroblasts, VPA also improved the efficiency of iPSC colony formation dramatically even without transduced Klf4 and c-Myc [48]. The effect of VPA on reprogramming efficiency suggests that VPA may influence a crucial step in turning somatic cells into iPSCs. Using the information found, we can infer that chromatin modifications, specifically histone acetylation status, play a critical role in reprogramming and that HDAC inhibitors can significantly improve reprogramming efficiency [48]. However, it is worth noting that many HDAC inhibitors identified so far 
have low specificity $[53,54]$. Moreover, each HDAC may be involved in multiple pathways. Therefore, it is important to search for more specific HDAC inhibitors and to understand the modes of action of each HDAC and HDAC inhibitor, in order to account for any unexpected side effects [53].

Recently, a simple and nonintegrating method for reprogramming has been developed by using synthetic mRNAs of the reprogramming factors, Oct4, Sox2, Klf4, c-Myc, and Lin28 [55]. This method allowed up to 36 -fold increase of reprogramming efficiency, compared to the retroviral approach of delivering reprogramming factors [55]. More recently, it has been shown that the expression of the microRNA cluster miR-302/367 allowed rapid and efficient reprogramming of both mouse and human somatic cells to iPSCs without exogenous transcription factors [56]. The development of transgene-free iPSCs with high efficiency may allow patient-specific regenerative medicine within grasp.

\section{Conclusions}

An emerging regulatory network controlling neural stem cell self-renewal and differentiation is defined by integration of epigenetic regulators with other cell-intrinsic regulators and cell-extrinsic signals from stem cell niches. Unraveling how HDACs function within this network to regulate neural stem cell self-renewal and neurogenesis is essential to better understand neural stem cell biology. It will facilitate the development of new and targeted therapies using neural stem cells for a host of neurological disorders, including neurodegenerative diseases, such as Alzheimer's and Parkinson's diseases, and brain injuries.

The success in iPSC derivation has brought the realization of the therapeutic potential of stem cell technology closer than ever to us. However, to reach the full potential of iPSC application, it will be essential to improve the methods for iPSC generation by avoiding viral integration and enhancing the reprogramming efficiency. The recent achievement in enhancing reprogramming efficiency using HDAC inhibitors may build a foundation for future studies by allowing scientists to delve deeper into understanding the complexity of epigenetics in reprogramming and to develop even more strategies to further improve reprogramming efficiency. With the ability to generate iPSCs with increased efficiency, a huge step is taken in the branch of regenerative medicine, in addition to disease modeling, and drug development. There is no doubt that iPSC technology will have a positive impact on stem cell therapies in the future.

\section{Acknowledgment}

The authors apologize to colleagues whose work could not be cited due to space limitations. Guoqiang Sun is Herbert Horvitz Postdoctoral Fellow. Chelsea Fu is supported by Eugene and Ruth Roberts Summer Student Program. This work is supported by NIH NINDS R01 NS059546 and RC1 NS068370 and California Institute for Regenerative Medicine Grant TR2-01832 (to Yanghong Shi).

\section{References}

[1] H. Wichterle and S. Przedborski, "What can pluripotent stem cells teach us about neurodegenerative diseases," Nature Neuroscience, vol. 13, no. 7, pp. 800-804, 2010.

[2] L. C. Cheng, M. Tavazoie, and F. Doetsch, "Stem cells: from epigeneticsto microRNAs," Neuron, vol. 46, no. 3, pp. 363-367, 2005.

[3] R. D. Hawkins, G. C. Hon, L. K. Lee et al., "Distinct epigenomic landscapes of pluripotent and lineage-committed human cells," Cell Stem Cell, vol. 6, no. 5, pp. 479-491, 2010.

[4] R. P. Koche, Z. D. Smith, M. Adli et al., "Reprogramming factor expression initiates widespread targeted chromatin remodeling," Cell Stem Cell, vol. 8, no. 1, pp. 96-105, 2011.

[5] Y. Shi, X. Zhao, J. Hsieh et al., "MicroRNA regulation of neural stem cells and neurogenesis," Journal of Neuroscience, vol. 30, no. 45, pp. 14931-14936, 2010.

[6] V. E. MacDonald and L. A. J. Howe, "Histone acetylation: where to go and how to get there," Epigenetics, vol. 4, no. 3, pp. 139-143, 2009.

[7] A. L. Clayton, C. A. Hazzalin, and L. C. Mahadevan, “ Enhanced histone acetylation and transcription: a dynamic perspective," Molecular Cell, vol. 23, no. 3, pp. 289-296, 2006.

[8] M. Haberland, R. L. Montgomery, and E. N. Olson, "The many roles of histone deacetylases in development and physiology: Implications for disease and therapy," Nature Reviews Genetics, vol. 10, no. 1, pp. 32-42, 2009.

[9] A. J. Ruthenburg, H. Li, D. J. Patel, and C. D. Allis, "Multivalent engagement of chromatin modifications by linked binding modules," Nature Reviews Molecular Cell Biology, vol. 8, no. 12, pp. 983-994, 2007.

[10] T. Kouzarides, "Acetylation: a regulatory modification to rival phosphorylation," EMBO Journal, vol. 19, no. 6, pp. 11761179, 2000.

[11] A. Alvarez-Buylla and S. Temple, "Stem cells in the developing and adult nervous system," Journal of Neurobiology, vol. 36, no. 2, pp. 105-110, 1998.

[12] F. H. Gage, G. Kempermann, T. D. Palmer, D. A. Peterson, and J. Ray, "Multipotent progenitor cells in the adult dentate gyrus," Journal of Neurobiology, vol. 36, no. 2, pp. 249-266, 1998.

[13] R. McKay, "Stem cells in the central nervous system," Science, vol. 276, no. 5309, pp. 66-71, 1997.

[14] S. Weiss and D. van der Kooy, "CNS stem cells: Where's the biology (a.k.a. beef)?” Journal of Neurobiology, vol. 36, no. 2, pp. 307-314, 1998.

[15] Y. Shi, G. Sun, C. Zhao, and R. Stewart, "Neural stem cell selfrenewal," Critical Reviews in Oncology/Hematology, vol. 65, no. 1, pp. 43-53, 2008.

[16] I. H. Park, R. Zhao, J. A. West et al., "Reprogramming of human somatic cells to pluripotency with defined factors," Nature, vol. 451, no. 7175, pp. 141-146, 2008.

[17] K. Takahashi, K. Tanabe, M. Ohnuki et al., "Induction of pluripotent stem cells from adult human fibroblasts by defined factors," Cell, vol. 131, no. 5, pp. 861-872, 2007.

[18] J. Yu, M. A. Vodyanik, K. Smuga-Otto et al., "Induced pluripotent stem cell lines derived from human somatic cells," Science, vol. 318, no. 5858, pp. 1917-1920, 2007.

[19] Y. Shi, "Induced pluripotent stem cells, new tools for drug discovery and new hope for stem cell therapies," Current Molecular Pharmacology, vol. 2, no. 1, pp. 15-18, 2009.

[20] S. Temple, "Stem cell plasticity-building the brain of our dreams," Nature Reviews Neuroscience, vol. 2, no. 7, pp. 513520, 2001. 
[21] G. Sun, R. T. Yu, R. M. Evans, and Y. Shi, "Orphan nuclear receptor TLX recruits histone deacetylases to repress transcription and regulate neural stem cell proliferation," Proceedings of the National Academy of Sciences of the United States of America, vol. 104, no. 39, pp. 15282-15287, 2007.

[22] J. L. MacDonald and A. J. Roskams, "Histone deacetylases 1 and 2 are expressed at distinct stages of neuro-glial development," Developmental Dynamics, vol. 237, no. 8, pp. 2256-2267, 2008.

[23] H. Liu, Q. Hu, A. Kaufman, A. J. D'Ercole, and P. Ye, "Developmental expression of histone deacetylase 11 in the murine brain," Journal of Neuroscience Research, vol. 86, no. 3, pp. 537-543, 2008.

[24] Y. Shi, C. D. Lie, P. Taupin et al., "Expression and function of orphan nuclear receptor TLX in adult neural stem cells," Nature, vol. 427, no. 6969, pp. 78-83, 2004.

[25] G. Zupkovitz, R. Grausenburger, R. Brunmeir et al., "The cyclin-dependent kinase inhibitor p21 is a crucial target for histone deacetylase 1 as a regulator of cellular proliferation," Molecular and Cellular Biology, vol. 30, no. 5, pp. 1171-1181, 2010.

[26] M. Ocker and R. Schneider-Stock, "Histone deacetylase inhibitors: signalling towards p21cip1/waf1," International Journal of Biochemistry and Cell Biology, vol. 39, no. 7-8, pp. 1367-1374, 2007.

[27] A. L. Gartel and A. L. Tyner, "Transcriptional regulation of the p21((WAF1/CIP1)) gene," Experimental Cell Research, vol. 246, no. 2, pp. 280-289, 1999.

[28] K. Nakano, T. Mizuno, Y. Sowa et al., "Butyrate activates the WAF1/Cip1 gene promoter through Sp1 sites in a p53-negative human colon cancer cell line," Journal of Biological Chemistry, vol. 272, no. 35, pp. 22199-22206, 1997.

[29] G. Lagger, A. Doetzlhofer, B. Schuettengruber et al., "The tumor suppressor p53 and histone deacetylase 1 are antagonistic regulators of the cyclin-dependent kinase inhibitor p21/WAF1/CIP1 gene," Molecular and Cellular Biology, vol. 23, no. 8, pp. 2669-2679, 2003.

[30] V. Balasubramaniyan, E. Boddeke, R. Bakels et al., "Effects of histone deacetylation inhibition on neuronal differentiation of embryonic mouse neural stem cells," Neuroscience, vol. 143, no. 4, pp. 939-951, 2006.

[31] Y. Hao, T. Creson, L. Zhang et al., "Mood stabilizer valproate promotes ERK pathway-dependent cortical neuronal growth and neurogenesis," Journal of Neuroscience, vol. 24, no. 29, pp. 6590-6599, 2004.

[32] J. Hsieh, K. Nakashima, T. Kuwabara, E. Mejia, and F. H. Gage, "Histone deacetylase inhibition-mediated neuronal differentiation of multipotent adult neural progenitor cells," Proceedings of the National Academy of Sciences of the United States of America, vol. 101, no. 47, pp. 16659-16664, 2004.

[33] F. A. Siebzehnrubl, R. Buslei, I. Y. Eyupoglu, S. Seufert, E. Hahnen, and I. Blumcke, "Histone deacetylase inhibitors increase neuronal differentiation in adult forebrain precursor cells," Experimental Brain Research, vol. 176, no. 4, pp. 672678, 2007.

[34] I. T. Yu, J. Y. Park, S. H. Kim, J. S. Lee, Y. S. Kim, and H. Son, "Valproic acid promotes neuronal differentiation by induction of proneural factors in association with $\mathrm{H} 4$ acetylation," Neuropharmacology, vol. 56, no. 2, pp. 473-480, 2009.

[35] G. Zupkovitz, J. Tischler, M. Posch et al., "Negative and positive regulation of gene expression by mouse histone deacetylase 1," Molecular and Cellular Biology, vol. 26, no. 21, pp. 7913-7928, 2006.
[36] R. L. Montgomery, J. Hsieh, A. C. Barbosa, J. A. Richardson, and E. N. Olson, "Histone deacetylases 1 and 2 control the progression of neural precursors to neurons during brain development," Proceedings of the National Academy of Sciences of the United States of America, vol. 106, no. 19, pp. 7876-7881, 2009.

[37] M. Jawerka, D. Colak, L. Dimou et al., "The specific role of histone deacetylase 2 in adult neurogenesis," Neuron Glia Biology, vol. 6, no. 2, pp. 93-107, 2010.

[38] D. M. Chuang, Y. Leng, Z. Marinova, H. J. Kim, and C. T. Chiu, "Multiple roles of HDAC inhibition in neurodegenerative conditions," Trends in Neurosciences, vol. 32, no. 11, pp. 591$601,2009$.

[39] C. L. Bowden, A. M. Brugger, A. C. Swann et al., "Efficacy of divalproex vs lithium and placebo in the treatment of mania. The Depakote Mania Study," Journal of the American Medical Association, vol. 271, no. 12, pp. 918-924, 1994.

[40] J. A. Thomson, J. Itskovitz-Eldor, S. S. Shapiro et al., "Embryonic stem cell lines derived from human blastocysts," Science, vol. 282, no. 5391, pp. 1145-1147, 1998.

[41] N. Maherali, R. Sridharan, W. Xie et al., "Directly reprogrammed fibroblasts show global epigenetic remodeling and widespread tissue contribution," Cell Stem Cell, vol. 1, no. 1, pp. 55-70, 2007.

[42] K. Okita, T. Ichisaka, and S. Yamanaka, "Generation of germline-competent induced pluripotent stem cells," Nature, vol. 448, no. 7151, pp. 313-317, 2007.

[43] K. Takahashi and S. Yamanaka, "Induction of pluripotent stem cells from mouse embryonic and adult fibroblast cultures by defined factors," Cell, vol. 126, no. 4, pp. 663-676, 2006.

[44] M. Wernig, A. Meissner, R. Foreman et al., "In vitro reprogramming of fibroblasts into a pluripotent ES-cell-like state," Nature, vol. 448, no. 7151, pp. 318-324, 2007.

[45] K. Hochedlinger and K. Plath, "Epigenetic reprogramming and induced pluripotency," Development, vol. 136, no. 4, pp. 509-523, 2009.

[46] E. Kiskinis and K. Eggan, "Progress toward the clinical application of patient-specific pluripotent stem cells," Journal of Clinical Investigation, vol. 120, no. 1, pp. 51-59, 2010.

[47] D. Huangfu, K. Osafune, R. Maehr et al., "Induction of pluripotent stem cells from primary human fibroblasts with only Oct4 and Sox2," Nature Biotechnology, vol. 26, no. 11, pp. 1269-1275, 2008.

[48] D. Huangfu, R. Maehr, W. Guo et al., "Induction of pluripotent stem cells by defined factors is greatly improved by smallmolecule compounds," Nature Biotechnology, vol. 26, no. 7, pp. 795-797, 2008.

[49] T. S. Mikkelsen, J. Hanna, X. Zhang et al., "Dissecting direct reprogramming through integrative genomic analysis," Nature, vol. 454, no. 7200, pp. 49-55, 2008.

[50] Y. Shi, J. T. Do, C. Desponts, H. S. Hahm, H. R. Schöler, and S. Ding, "A combined chemical and genetic approach for the generation of induced pluripotent stem cells," Cell Stem Cell, vol. 2, no. 6, pp. 525-528, 2008.

[51] S. Kishigami, E. Mizutani, H. Ohta et al., "Significant improvement of mouse cloning technique by treatment with trichostatin A after somatic nuclear transfer," Biochemical and Biophysical Research Communications, vol. 340, no. 1, pp. 183189, 2006.

[52] A. Rybouchkin, Y. Kato, and Y. Tsunoda, "Role of histone acetylation in reprogramming of somatic nuclei following nuclear transfer," Biology of Reproduction, vol. 74, no. 6, pp. 1083-1089, 2006. 
[53] J. L. MacDonald and A. J. Roskams, "Epigenetic regulation of nervous system development by DNA methylation and histone deacetylation," Progress in Neurobiology, vol. 88, no. 3, pp. 170-183, 2009.

[54] A. Villar-Garea and M. Esteller, "Histone deacetylase inhibitors: understanding a new wave of anticancer agents," International Journal of Cancer, vol. 112, no. 2, pp. 171-178, 2004.

[55] L. Warren, P. D. Manos, T. Ahfeldt et al., "Highly efficient reprogramming to pluripotency and directed differentiation of human cells with synthetic modified mRNA," Cell Stem Cell, vol. 7, no. 5, pp. 618-630, 2010.

[56] F. Anokye-Danso, C. M. Trivedi, D. Juhr et al., "Highly efficient miRNA-mediated reprogramming of mouse and human somatic cells to pluripotency," Cell Stem Cell, vol. 8, no. 4, pp. 376-388, 2011. 

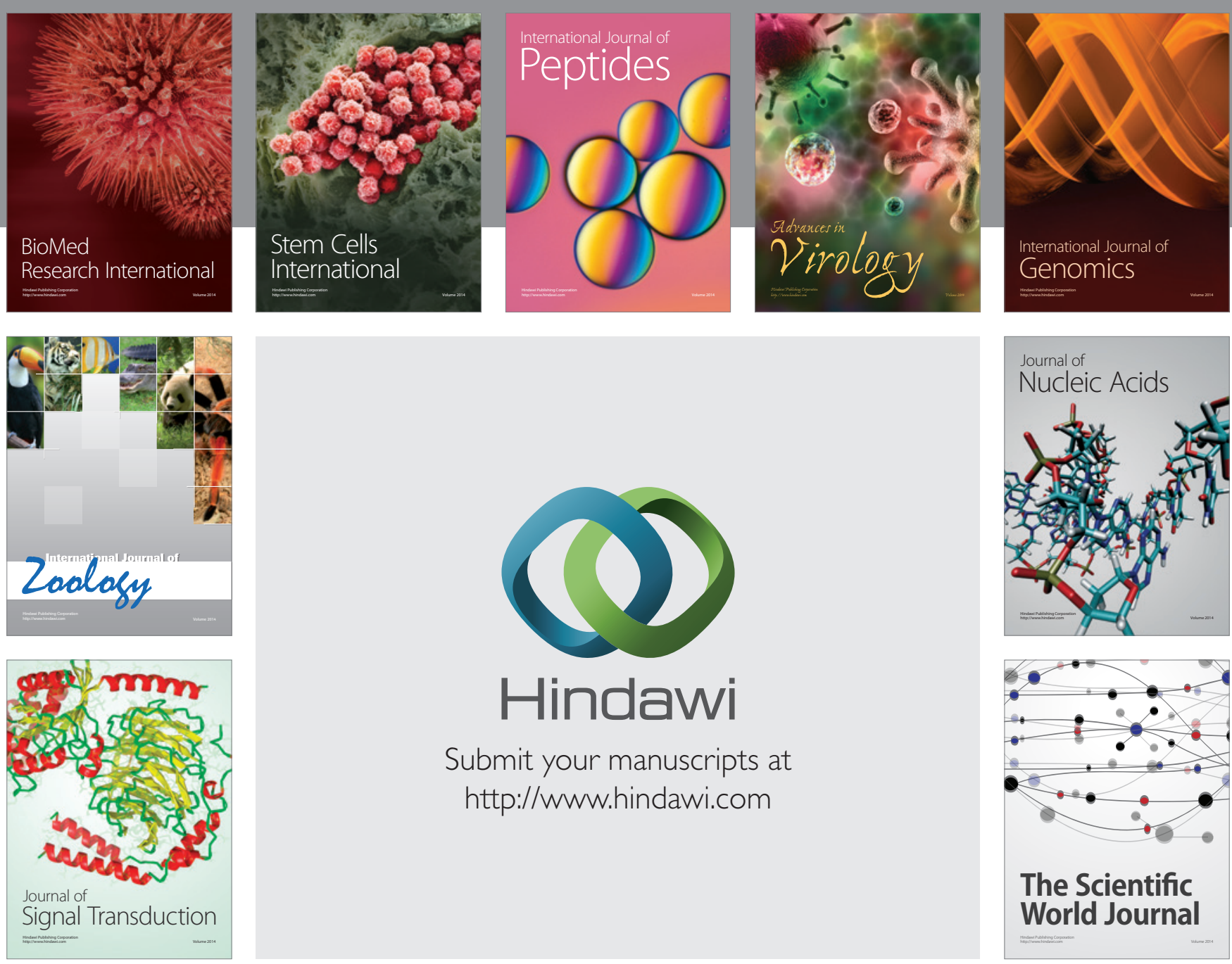

Submit your manuscripts at

http://www.hindawi.com
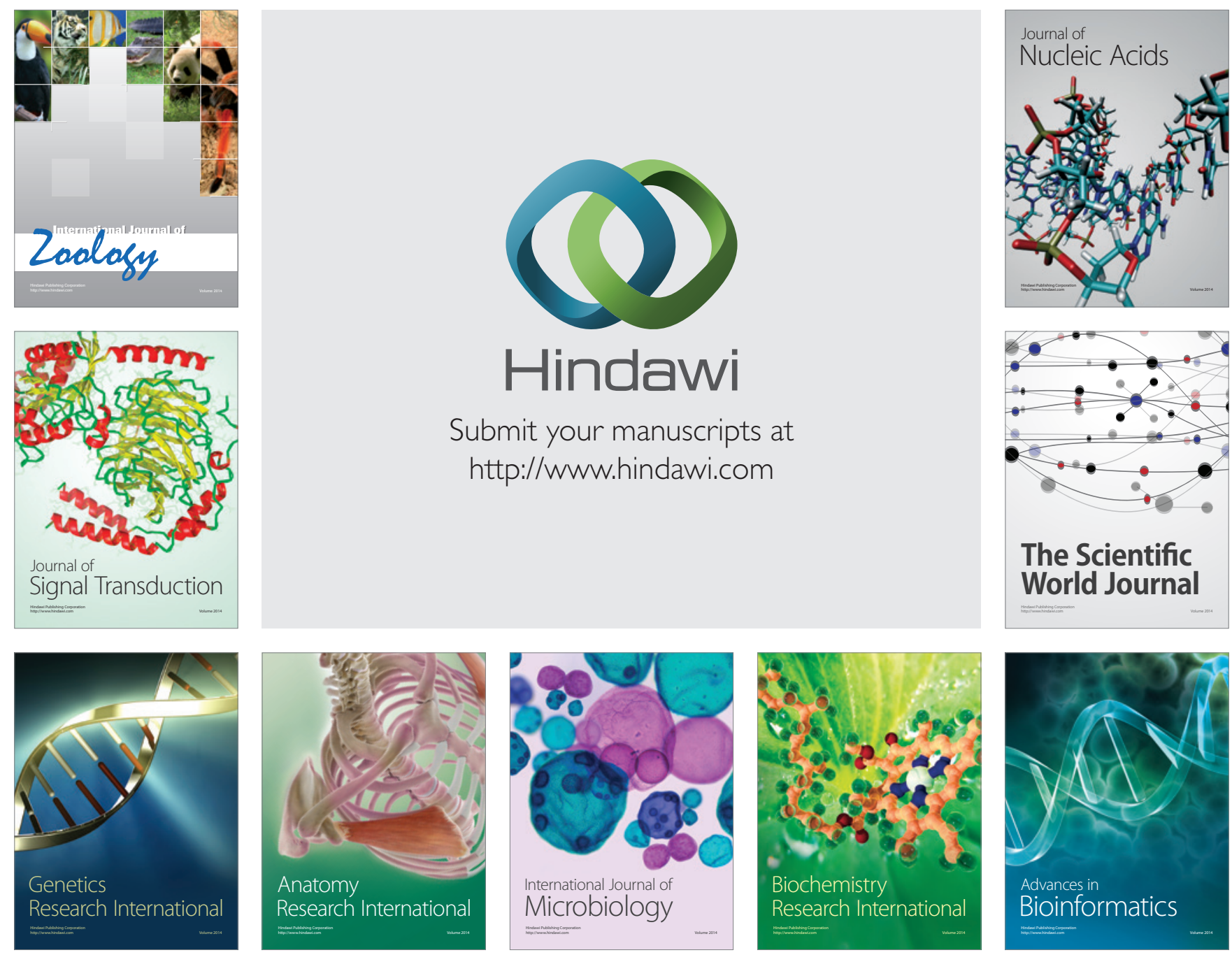

The Scientific World Journal
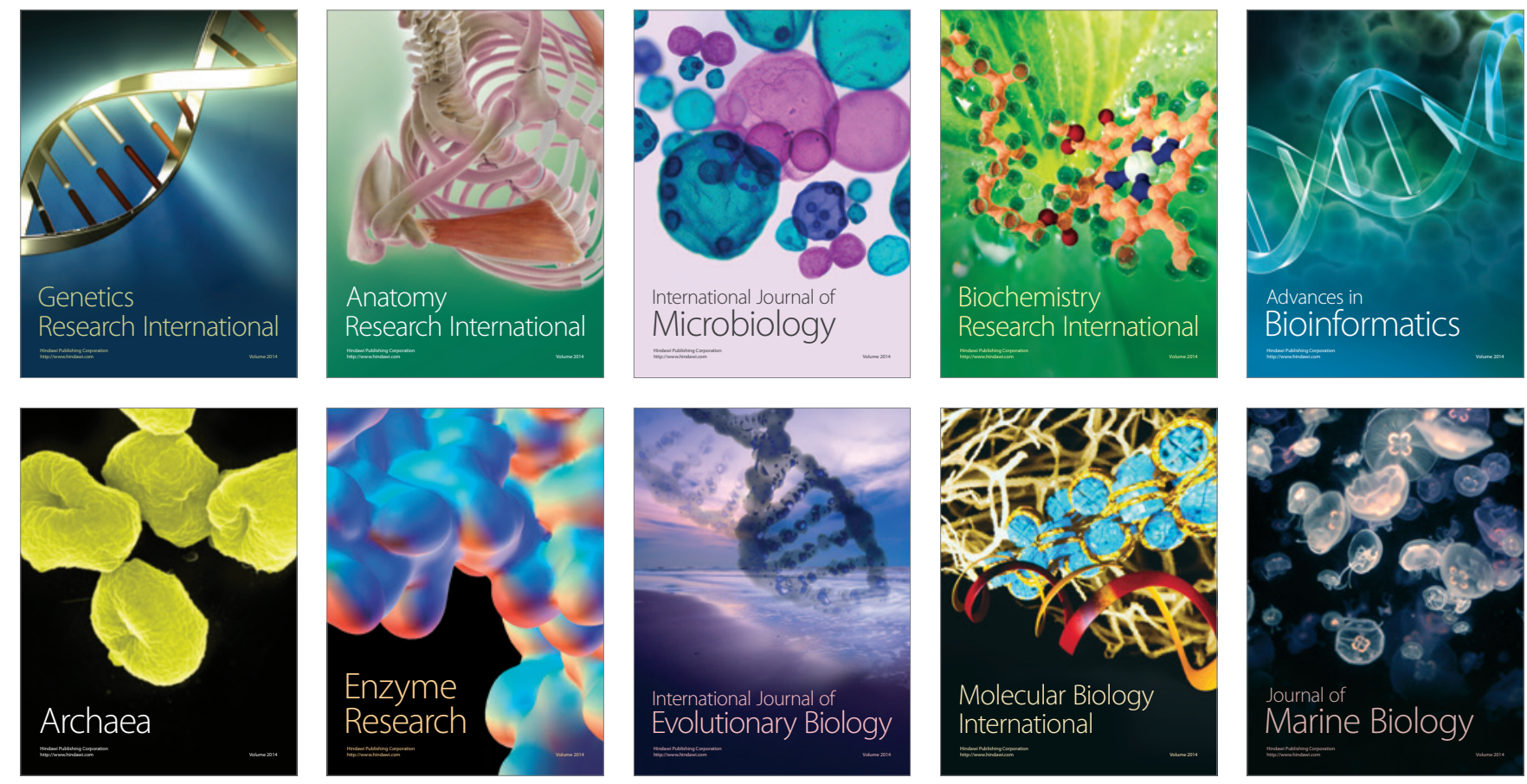